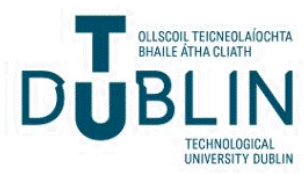

Technological University Dublin

ARROW@TU Dublin

\section{Towards 3D in vitro models for the study of cardiovascular tissues and disease}

Claire M. Brougham

Technological University Dublin, claire.brougham@tudublin.ie

Alan J. Ryan

Royal College of Surgeons in Ireland

Carolina D. Garciarena

Royal College of Surgeons in Ireland

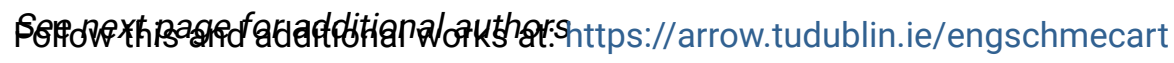

Part of the Biology Commons, Circulatory and Respiratory Physiology Commons, and the Medical Biotechnology Commons

\section{Recommended Citation}

Brougham, C.M.*et al. (2016) Towards 3D in vitro models for the study of cardiovascular tissues and disease, Drug Discovery Today 2016; 21; 9; 1437-1445. https://doi.org/10.1016/j.drudis.2016.04.014

This Article is brought to you for free and open access by the School of Mechanical and Design Engineering at ARROW@TU Dublin. It has been accepted for inclusion in Articles by an authorized administrator of ARROW@TU Dublin. For more information, please contact arrow.admin@tudublin.ie, aisling.coyne@tudublin.ie, gerard.connolly@tudublin.ie.

Funder: This work was supported by the Irish Heart Foundation (grant number 1045940) to FJO'B and CMB, the European Research Council European Community's Seventh Framework Programme (FP7/2007-2013) to FJO'B and the Science Foundation Ireland Career Development Award (grant number 13/CDA/2119) to SWK. 
Authors

Claire M. Brougham, Alan J. Ryan, Carolina D. Garciarena, Steve W. Kerrigan, and Fergal J. O'Brien

This article is available at ARROW@TU Dublin: https://arrow.tudublin.ie/engschmecart/54 


\section{Towards 3D in vitro models for the study of cardiovascular tissues and disease}

\section{Alan J. Ryan ${ }^{1,2,3,6}$, Claire M. Brougham ${ }^{1,2,4,6}$, Carolina D. Garciarena ${ }^{5}$, Steven W. Kerrigan ${ }^{5}$ and Fergal J. O'Brien ${ }^{1,2,3}$}

\footnotetext{
${ }^{1}$ Tissue Engineering Research Group, Department of Anatomy, Royal College of Surgeons in Ireland, 123 St Stephen's Green, Dublin 2, Ireland

${ }^{2}$ Trinity Centre for Bioengineering, Trinity Biomedical Sciences Institute, Trinity College Dublin, 152-160 Pearse Street, Dublin 2, Ireland

${ }^{3}$ Advanced Materials and Bioengineering Research (AMBER) Centre, Royal College of Surgeons in Ireland and Trinity College Dublin, Dublin, Ireland

${ }^{4}$ School of Mechanical and Design Engineering, Dublin Institute of Technology, Bolton Street, Dublin 1, Ireland

${ }^{5}$ Cardiovascular Infection Research Group, School of Pharmacy \& Molecular and Cellular Therapeutics, Royal College of Surgeons in Ireland, 123 St Stephen's Green, Dublin 2, Ireland
}

\section{The field of tissue engineering is developing biomimetic biomaterial scaffolds that are showing} increasing therapeutic potential for the repair of cardiovascular tissues. However, a major opportunity exists to use them as 3D in vitro models for the study of cardiovascular tissues and disease in addition to drug development and testing. These in vitro models can span the gap between 2D culture and in vivo testing, thus reducing the cost, time, and ethical burden of current approaches. Here, we outline the progress to date and the requirements for the development of ideal in vitro 3D models for blood vessels, heart valves, and myocardial tissue.

\section{Introduction}

Cardiovascular disease (CVD) represents a major healthcare and economic burden and is currently the leading cause of noncommunicable deaths worldwide [1,2]. The diseases of hypertension, heart failure, cardiac hypertrophy, cardiomyopathy, endocarditis, thrombosis, atherosclerosis, valvular regurgitation, and stenosis resulted in US $\$ 320$ billion expenditure in healthcare costs in 2011 in the USA alone [2]. Despite the staggering annual mortality of 17.5 million in 2012, the number of deaths has steadily declined since the 1970s as a result of scientific advances [1,3]. Increased funding for research in the cardiovascular field has been vital to decelerate this progression and, with an aging population, this research needs to continue to discover and develop new therapeutics, medical devices, bioengineered replacement tissues, and surgical solutions.

Research into CVD is typically carried out using 2D in vitro culture conditions that, although relatively cheap and simple, are a non-ideal environment to study cellular phenotype and

Corresponding author: O'Brien, F.J. (fjobrien@rcsi.ie)

${ }^{6}$ These authors contributed equally to this work. behaviour (Fig. 1) [4,5]. The benefit of in vitro 3D models is their improved physiological relevance and, although imperfect, they could serve as a bridge between 2D cultures and in vivo models, to determine the mechanism and efficacy of targeted formulations and devices [5]. While preclinical animal models continue to serve as a key efficacy and safety requirement for the translation of therapeutics, the degree of reliability achieved in reproducing human pathophysiology in animal models is frequently inadequate [6]. This undoubtedly contributes to the low success rate in developing efficient and safe therapies [7,8]. Additionally, in recent years, we have witnessed an increased awareness of the ethical implications of the use of animals for medical research, encouraged by funding bodies and editors, and represented by the concepts of the 3Rs (replacement, reduction, and refinement) principle. These limitations in the use of both animal models and classical in vitro 2D models have propelled the expansion of research into new in vitro 3D cardiovascular models that allow for greater control of variables and also a shortened experimental time compared with in vivo models [9]. Ideal 3D models need to replicate not only the anatomical macrostructure, but also the tissue and cell-level microstructures, including appropriate cell types, 
(a)

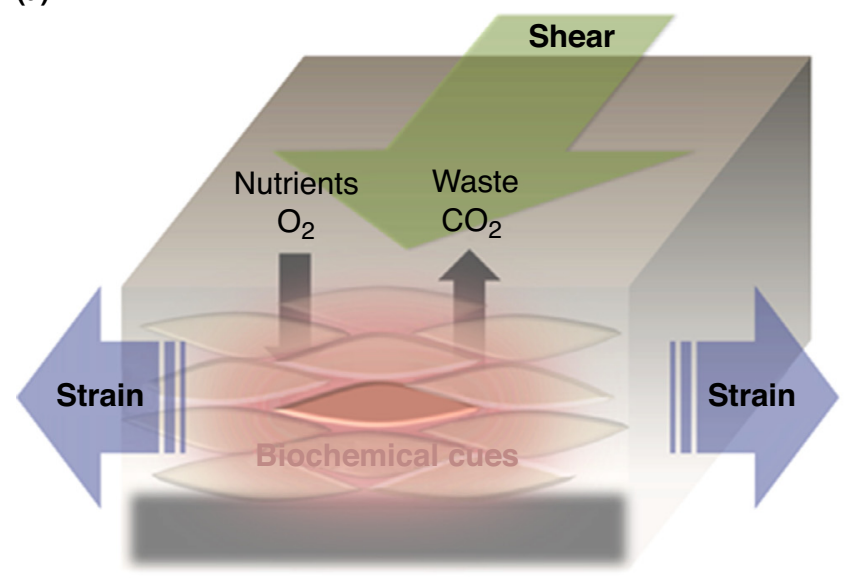

(c)

(d) Cardiomyocyte neonatal

$\mathrm{Oh}$

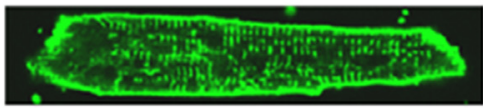

$24 \mathrm{~h}$

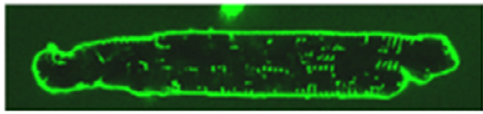

$72 \mathrm{~h}$

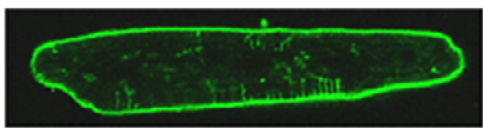

(b)

Adult cardiomyocytes

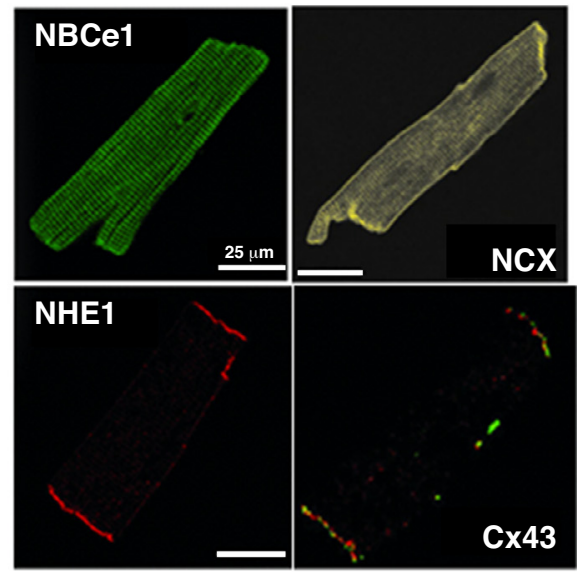

(e)

3D culture (strain/shear) neonatal cardiomyocytes

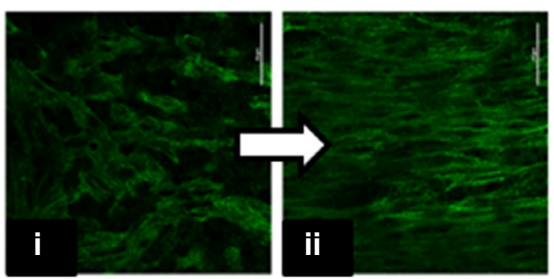

Endothelial cells

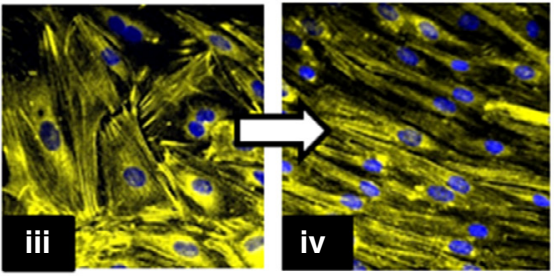

Drug Discovery Today

\section{FIGURE}

Importance of the microenvironment to cardiovascular cellular phenotypes. (a) Biomechanical influences within the 3D microenvironment of the cell affect the cellular phenotype. (b) This is illustrated within native adult cardiomyocytes by the selective localisation and/or function of their cellular proteins, which maintain cell structure, intercellular communications, intracellular $\mathrm{pH}$ regulation, and $\mathrm{Ca}^{2+}$ handling. (c) Conventional static $2 \mathrm{D}$ culture of adult myocytes fails to preserve the adult phenotype and (d) induces progressive dedifferentiation to a foetal phenotype. (e) The functional phenotype can be rescued, for example, by replicating the physiological strain in cultured rat neonatal cardiomyocytes (i,ii) or by application of shear stress on cultured human aortic endothelial cells (iii,iv). Reproduced, with permission, from [10] (b), [11], (c), [12] (d), and [13,14] (e).

biomimetic extracellular matrix (ECM) arrangement, and appropriate physiological cues. This will ensure greater precision and reliability for translation, while reducing the number of animals used in development (Fig. 1).

Although preclinical animal models are a necessary step towards the regulatory approval of new devices and pharmaceutics, in vitro testing can reduce translation costs and time and help to identify the mechanism of action together with any associated risks. Although preclinical drug screening is typically achieved with mouse models, the human cardiac physiological response to drugs has been shown to be markedly different to that in mice, leading to relatively high failure rates during the clinical trial phase. Translating a drug from a US Food and Drug Administration (FDA)-classed new molecular entity (NME) to approved clinical product is estimated to cost between US $\$ 0.8$ billion and US\$1.8 billion and typically spans $8-12$ years [15], not including the initial years of investment in basic research leading to initial drug discovery. Little is known about the safety and offtarget effects of NMEs before human clinical trials resulting from phylogenetic differences between humans and animal models. This has resulted in the catastrophic failure of many Phase I drug trials and further highlights the inadequacies of current in vitro testing, which often are not representative of the in vivo preclinical environment, let alone the clinical situation in humans. Along similar lines, medical device development is estimated to cost between US\$31 million and US\$94 million (depending on device risk) before clinical testing [16] and, although the unwanted effects of devices tend to be more mechanical, combination products still need a representative in vitro testing platform. Indeed, simpler medical devices can also benefit from an improved in vitro testing environment, where device long-term performance, and biocompatibility testing can be tested in a more 
characteristic way, while being fully controllable, accessible, and measurable.

The rapid expansion of the field of cardiovascular tissue engineering has seen the development of biomaterials and neotissues with the innate capacity to grow and remodel in vitro and, as such, can be utilised as platforms to assess pharmaceutics and devices before preclinical work. Currently used in vitro 3D models include spheroids, hydrogels, ECM-derived matrices, scaffolds, and decellularised tissues and/or organs [5]. For the treatment of CVD, tissue engineering has focussed on bioengineered vessels, valves, and regenerated myocardial tissue, with the additional potential to enhance their regenerative capacity through the incorporation of drugs, genetic tools, and cells. Thus, biomaterials for tissue engineering provide templates upon which multiple cell types can be cultured, and can combine genes [17] and growth factors together with ECM constituents $[18,19]$. Mechanical and architectural properties of biomaterials can be easily refined, from varying their stiffness to their porosity and pore size [18,20,21]. In addition, incorporation of surface-binding ligands and antibodies can encourage specific cell attachment, while dynamic forces can be applied to these biomaterials, to elucidate responses also observed in vivo [22]. The toolset available to bioengineers has now reached a point where the in vitro replication of native tissues is achievable through innovative biofabrication techniques, advanced cell manipulation, and the application of physiologically relevant environments through bioreactor technologies.
Here, we focus on three examples of potential 3D models of cardiovascular tissue: vascular grafts, cardiac valves, and myocardial tissue. It is envisioned that these 3D models will provide test beds for advanced drug and device testing in addition to disease modelling and precision medicine screening [22]. We outline the parameters that should be considered in developing a true in vitro model for each tissue and describe recent advances in each area.

\section{D in vitro models of blood vessels}

Tissue-engineered blood vessels (TEBVs) hold great promise clinically, where they could be utilised for coronary artery bypassing, peripheral artery disease, or as an arteriovenous graft for haemodialysis access [23]. Strides towards achieving these goals have been made using several distinct approaches to TEBV development with both autologous [24] and allogeneic cells [23,25], and matured through the application of physiological haemodynamic forces within pulsatile bioreactors. The native blood vessel structure comprises a multi-lamellar architecture with a distinct arrangement of endothelial cells (ECs), smooth muscle cells (SMCs), and fibroblasts, which together maintain a normal functional vessel. Replication of native vessels can be achieved by generating biomimetic scaffolds comprising native polymers, such as collagen and elastin [18], in a complex 3D tubular architecture [26], and the correct spatial arrangement of vascular cells [27] (Fig. 2a,b). Interestingly, the commercial approach for implantable TEBVs by some of the leading companies in the field, including Humacyte (a)

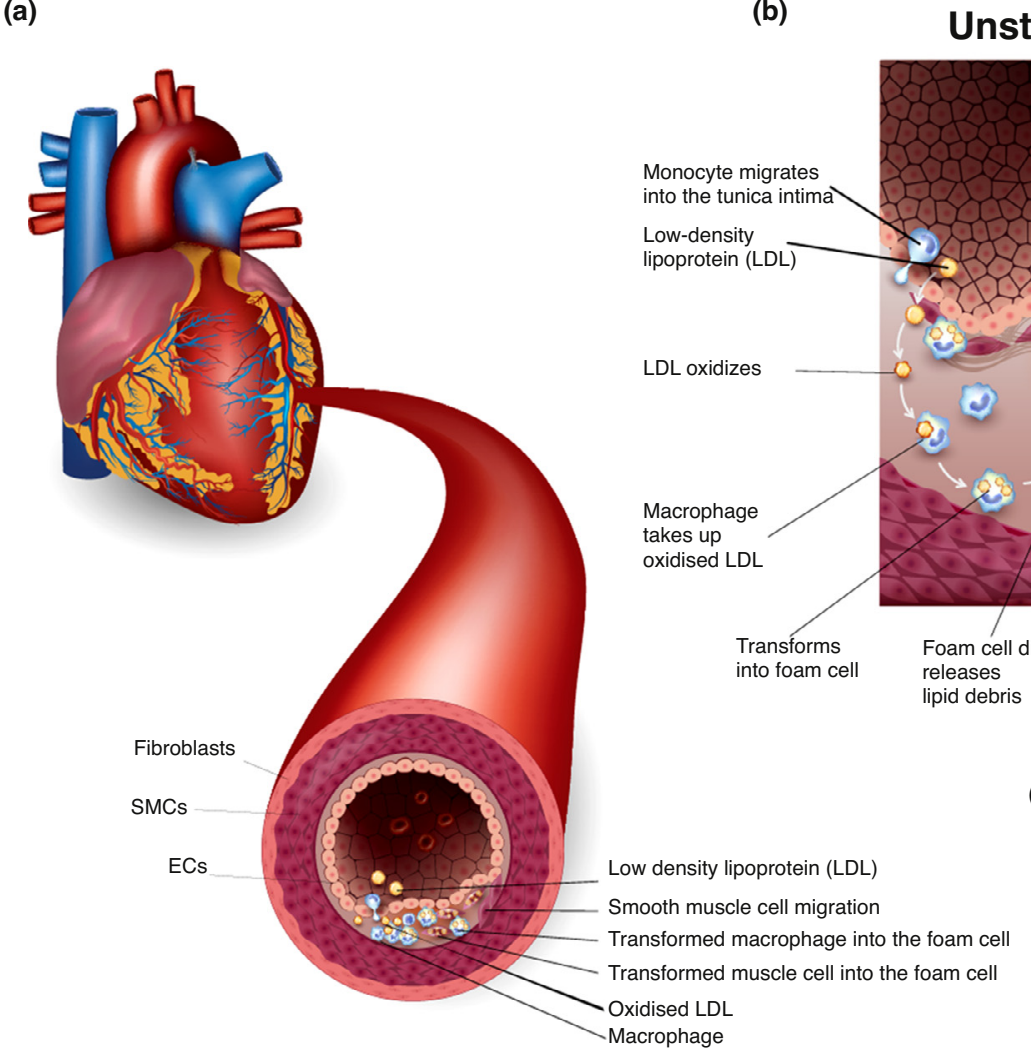

FIGURE 2

Atherosclerotic disease progression and in vitro device testing. The healthy vessel wall (a) is infiltrated by macrophages, which begin to form foam cells and initiate the degenerative cascade, which eventually results in an unstable atherosclerotic lesion (b). The complex process of atherosclerotic lesion progression has proven difficult to replicate in vitro. Radiographic image of a stent deployed within a blood vessel mimic (v) [28]. Abbreviations: EC, endothelial cells; SMC, smooth muscle cells. 
Inc. and Cytograft Tissue Engineering Inc, has progressed towards growing TEBVs in vitro and then decellularising these TEBVs to reduce costs and complexity, and to ease the regulatory pathway. The advances made towards the realisation of a clinically available vascular graft have left the field with the knowledge and toolsets required to generate high-fidelity vascular analogues in vitro that could also be used preclinically in vitro.

One of the initial areas of interest for in vitro TEBV models was for pharmacological studies. Nicolas L'Heureux, one of the pioneers of TEBVs, demonstrated in 1998 the ability to create triplelayered grafts [28], before generating individual vascular tissue layers for pharmacological studies of contraction [29]. These medial layer equivalents were generated from human vascular SMCs and were shown to be responsive to multiple vasoconstrictors (e.g. histamine, ATP, bradykinin, and UTP) in a concentration-dependent manner. This reduction in complexity for a pharmacological study was an interesting approach because it allowed the physiological response of a single cell type and/or tissue layer to be determined. A similar approach was utilised to create medial layer-equivalent TEBVs that maintained the subtle differences in receptor expression and response to endothelin as the native vessels from which the cells were extracted [30].

The innermost layer of blood vessels, the tunica intima, is lined with ECs, a vital component to maintain haemostasis and control vascular permeability. However, the deployment of intravascular devices, such as stents, is known to damage the EC lining of vessels and so all the device deployment studies to date have first focused on generating a model vessel containing a confluent EC monolayer [31-33] (Fig. 2c). While these studies used EC monolayers that were preconditioned via a bioreactor flow loop system to simulate in vivo haemodynamics, the underlying vessels utilised thus far for device testing have been of a synthetic nature, such as expanded polytetrafluoroethylene [31,32] or silicone [33]. Consequently, there exists a need for an in vitro TEBV model that can not only simulate EC damage during device deployment, but also contains a SMC-seeded tunica media (middle layer) to simulate restenosis, the narrowing of vessels, which is commonly caused by neointimal hyperplasia of SMCs because of device deployment [34].

In conjunction with device testing, the use of a TEBV as an in vitro model might also be a useful platform to study CVDs, such as atherosclerosis, which alone is responsible for $67.8 \%$ of CVDrelated deaths [35]. Although our ability to combat the clinical manifestations of atherosclerosis with advanced treatment modalities has resulted in reduced disease mortality [36], the prevention and reversal of the disease itself remains unfulfilled. Atherosclerosis is a complicated disease with the pathogenesis involving ECs, SMCs, lipoproteins, and macrophages over a time period often spanning decades (Fig. 2a,b). Early in vitro models of the disease utilised permeable membranes for the co-culture of ECs and SMCs, and successfully demonstrated the transmigration of monocytes across the EC layer and uptake of low-density lipoprotein (LDL) to form foam cells [37]. Although these were important steps in the field, they do not accurately represent the 3D environment of native vasculature cells. Some progress towards a biomimicking atherosclerotic model was made with the creation of flat 3D fibrin gels, which were capable of long-term culture and facilitated the formation of foam cells [38]. Utilising this model enabled examination of LDL-induced expression of interleukin 8 , a neutrophil chemokine, by SMCs and the subsequent recruitment of neutrophils into the subendothelial space, a previously unproven association [39].

An additional important factor in the progression of atherosclerosis is the effect of flow-induced shear stress, and cyclical strain [40]. A 3D tubular architecture is required to impart these forces accurately, in conjunction with pulsatile flow bioreactors, which can control the fluid forces applied. Such a model has been shown to be capable of generating the first 3D tubular TEBV, which mimicked the early stages of atherosclerosis under dynamic culture conditions [41]. With few research groups seemingly tackling the issue of creating high-fidelity atherosclerotic disease models, the progress in the field has understandably been slow to date. Furthermore, because vulnerable plaque lesions take decades to form naturally in vivo, the next step in this research must be to drastically accelerate the lesion-forming process in vitro (Fig. 2b) to allow for analysis of the parameters that affect the progression of this disease. Having a representative 3D model of atherosclerosis would allow the in vitro testing of therapeutics that could directly target lesions in a controlled and measurable way. In fact, the targeting of atherosclerotic lesions via nanoparticles has recently been modelled in vitro with a microfluidic system containing an atherosclerotic endothelium with controlled permeability, opening the field to further use of these models for modelling targeted drug delivery [42]. Transferring this type of testing into a larger 3D in vitro model is possible using the techniques developed for TEBVs.

Although great advances have been made in the field towards realising the use of TEBVs as implantable grafts to replace diseased or damaged tissue, the tissue complexity achieved is far beyond that utilised for device testing and disease modelling. This highlights the disconnect between the in vitro models generated, which display poor physiological relevance, and those that the field is undoubtedly capable of generating for use as implantable TEBVs.

\section{D in vitro models of heart valves}

With an increasing aging population and no proven pharmaceutical therapies to decelerate it, valvular disease is predicted to become the next global epidemic [43], with a prevalence of disease observed in aortic and mitral valves. A representative in vitro 3D model of aortic heart valves would help elucidate the mechanisms driving calcific aortic valvular disease (CAVD), a disease that affects $2.8 \%$ of the population [2], or endocarditis (bacterial and viral), both of which have limited treatment options [44]. Mitral valves also have calcification of their chordae tendineae, annulus, and the valve leaflets, requiring valve repair or replacement. Tissue-engineering strategies for heart valves are varied, with a multitude of cell sources [45], scaffold materials [19], and fabrication strategies currently under investigation [46-50]. However, for a tissue-engineered valve to serve as an in vitro 3D model, ECMderived scaffold materials and native valvular cells would be a requisite starting point, followed by biomimetic ECM structure and appropriate mechanical properties. Many tissue-engineered valves utilise cell sources such as umbilical vein cells and mesenchymal stem cells [45], cell types that would be suboptimal in an in vitro 3D model of the valve, but have advantages for engineered autologous tissues because of their ability to differentiate and act as valvular-type cells. However, many of the sophisticated valve 
scaffolds that have been developed through tissue engineering could be easily populated with more representative cells for an in vitro model. Here, we concentrate on the aortic valve, because most tissue-engineering strategies that could be used as in vitro models are focussed on this structure.

The highly organised configuration of the aortic valvular ECM prevents regurgitation of blood through coaptation of its three cusps or leaflets, each of which has the same trilayered microstructure. The fibrosa, spongiosa, and ventricularis layers occupy an overall thickness of approximately $1 \mathrm{~mm}$ and comprise collagen, elastin, and glycosaminoglycans in a specific arrangement (Fig. 3). Valvular interstitial cells (VICs) occupy the tissue layers, maintaining valve homeostasis through regulation of cellular proliferation and tissue remodelling. Although VICs are a fibroblastic cell type, they have five different phenotypes, and can convert from one form to another [51]. The nonthrombogenic interface of the valves between the blood and VICs is maintained by valvular endothelial cells (VECs). At a basic structural and functional level, the valvular and vascular endothelium appear similar; however, comparisons have identified hundreds of significantly different genes [52]. In dynamic culture, their response to shear and pressure is different and their transcriptional gene expression profile also differs when exposed to the same mechanical environment [52-54]. VECs regulate the activation of VICs through mechanotransduction, nitric oxide, vasodilatory prostanoids, and endothelin 1 [51].

In vitro, individual cellular components of the complex valve structure have been examined in isolation using 3D models, with an aim of understanding the mechanisms driving CAVD. The expression of growth factors by VICs has been examined at the edge of a damaged VIC layer using an 'in vitro wound model' [55], advancing understanding of VICs in a simple model system. 3D structures, such as PEG hydrogels, have been used to characterise VICs in isolation [56,57] and the reaction of VIC to substrate stiffness [57-60]. However, without the interactions of VECs within the correct ECM structure, these 3D models are limited.

The creation of a layered co-culture system with the physiological ECM components and structure is challenging, although simple VIC/VEC co-culture studies have been carried out. An interesting method using magnetic levitation was proposed by Grande-Allen's group [63], who also proposed a synthetic PEG hydrogel system to encourage layer-specific cell growth [64]. Collagen gels incorporating glycosaminoglycans have been used in both single cell cultures and co-cultures to investigate the interactions of ECM proteins with cells [65-68]. However, without the correct ECM structure found in vivo, the addition of these matrix components facilitates limited learning, because their structure, mechanical properties, and orientation are not considered.

The introduction of dynamic culture is a necessary parameter to investigate cardiovascular cell interactions, but further complicates any experimental setup and, as such, limited models have been developed to date. Collagen hydrogels have been used to assess VIC response to cyclic strain [69] and VIC/VEC response to shear stress $[70,71]$ but again, because of the lack of a biomimetic ECM structure, these studies are limited. Other dynamic studies focussed on the development of tissue-engineered valve replacements; however, these studies have not utilised VICs [72] because, for tissue-engineered valves, this cell source would be unavailable when developing valves for patients. However, there is the potential to use these scaffold arrangements to study VIC-VEC interactions in dynamic culture. A porous fibrin-collagen-glycosaminoglycan scaffold was recently developed [20] that shows potential for use as a platform to examine VIC-VEC interactions in dynamic culture. This model system, created from natural materials, has mechanical properties that can be altered as per experimental needs, can be formed into any shape, used within a dynamic environment, and can facilitate homogenous VIC distribution while maintaining a monolayer of VECs [73].

CAVD pathogenesis is far from fully understood; it is hypothesised to result from both injury to the valve and the subsequent activation of cells to repair the injury, while also involving the reactivation of developmental processes, such as the endothelialto-mesenchymal transformation (EMT) [74]. A complete understanding of CAVD will allow for the development of suitable smallmolecule/drug treatment options for this disease. Representative $3 \mathrm{D}$ models of the aortic valve can elucidate that knowledge

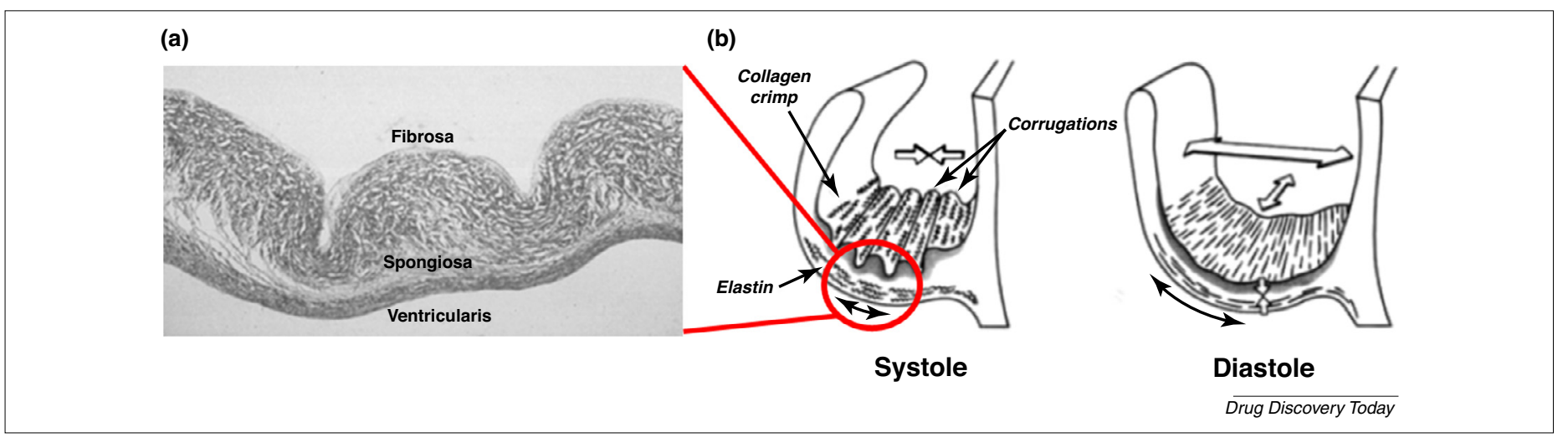

\section{FIGURE 3}

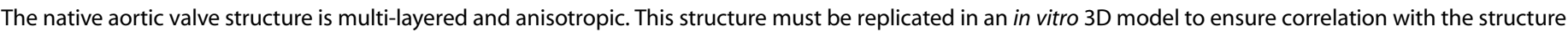

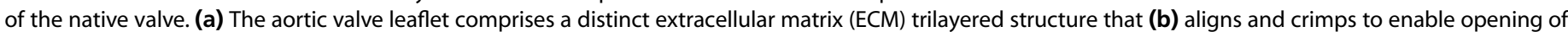

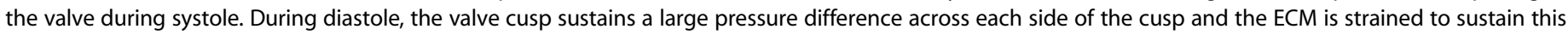

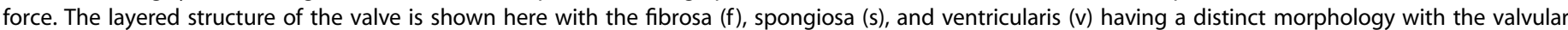
endothelial cells covering the ventricular and aortic side of the valve.

Reproduced, with permission, from [61,62]. 
through the identification and understanding of cellular and ECM changes in early disease states. This could lead to use of 3D models for the controlled, advance screening of various treatments to address the changes seen in the diseased state. These treatments might range from small-molecule/drug treatments to devices, and a suitable 3D model would be an invaluable tool for assessment of these innovations. Initial observations on individual cell layers have shown interactions between serotonergic medications and the development of valvular heart disease [75]. Similarly, the mutations in the signalling and transcriptional regulator $\mathrm{NOTCH}$ 1 have been widely investigated because of the association between NOTCH 1 and a range of developmental aortic valve anomalies $[76,77]$. Although many of these developmental studies are performed in vivo, the use of an in vitro 3D model with correct $\mathrm{ECM}$ architecture and human cells could progress knowledge and lead to the identification of a treatment for CAVD. Modification of the geometry used can allow optimisation for pulmonary, mitral, and tricuspid valves, enabling examination of more individual valvespecific conditions.

Given the distinctiveness of valvular cells, the architecture of the valve itself, and the haemodynamic forces experienced by the tissue, a complete 3D model of the aortic valve can only be found in vivo, and explanted human and porcine valves have been studied to understand cellular dysfunction in both aortic and mitral valves $[78,79]$. The next step is to have a representative in vitro $3 \mathrm{D}$ model to facilitate research into the causes and treatment of valve calcification, which would accelerate progress in combating this increasingly prevalent disease.

\section{D in vitro models of myocardial tissue}

Modelling of cardiac tissue response in vitro is important because it enables the examination of CVD and potential drug responses before progressing to costly clinical trials. The myocardium is involved in the propagation of electrical signals and also in force generation through the cardiomyocyte-rich tissue and so can be considered a functional unit of the heart wall. Clinical manifestations of myocardial loss and/or dysfunction are traditionally observed after ischaemic events, such as myocardial infarction, resulting in the loss of functionally viable myocardium, which can lead to the downward spiral to heart failure.

2D monolayers of cardiomyocytes have been used successfully to estimate drug-induced apoptosis and cardiac tissue damage and/or breakdown (troponin and lactate dehydrogenase release) [80]. However, these studies have been performed using static cultures of immature, neonatal cardiomyocytes, which are not representative of the adult, fully differentiated, cell. In addition, it is widely known that adult cardiomyocytes dedifferentiate and do not survive medium-term culture (Fig. 1). Moreover, acquired or inherited cardiomyopathies are often characterised by impaired functional characteristics, with cardiomyocyte contractile forces and kinetics helping to determine final ejection fractions. Therefore, 3D myocardium models are an important toolset with which to determine the mechanism of action of cardiac drugs, potential pro-arrhythmic effects of drugs, protein knockdown studies, in conjunction with heart disease modelling.

Tiburcy et al. [81] showed that 3D culture can recapitulate suitable environmental conditions to model hypertrophic cardiac growth by inducing terminal differentiation of neonatal rat cardiomyocytes and remodelling of a collagen type I-based hydrogel. Further advancement of collagen hydrogel-based models has been achieved by moulding microtissues around microfabricated silicone posts (Fig. 4a,b), where the tissue contraction force generation can then be determined through the deflection of the silicone posts [82]. Although these studies utilised rat neonatal cardiomyocytes, a more promising approach might be the creation of a human pluripotent stem cell-based model to generate adult human cardiomyocytes. Thavandiran et al. [83] reported the ability to create cardiac microtissues using human embryonic stem cells, (a)
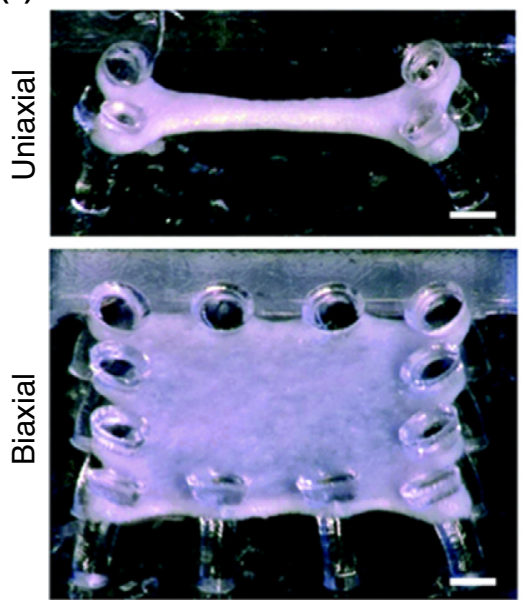

(b)
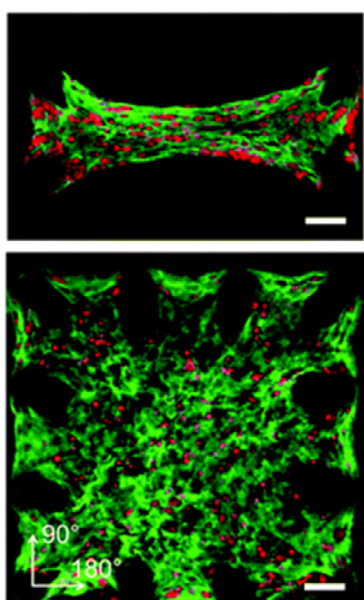

(c)

Simulated stress

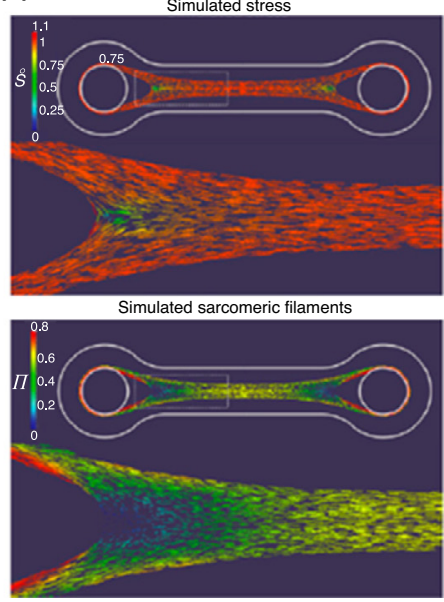

Drug Discovery Today

\section{FIGURE 4}

Engineering functional cardiac microtissues and simulation stress-induced maturation. (a) Engineered cardiac microtissues comprising neonatal mouse cardiomyocytes in a collagen I hydrogel that was contracted around polydimethylsiloxane microposts in a uniaxial or biaxial configuration. (b) Collagen (green) and cell (red) orientation during maturation was then tracked via fluorescent probes. (c) The stress-induced alignment and maturation of these cardiac microtissues can also be accurately simulated to inform future design considerations for these tissues.

Adapted, with permission, from $[83,86]$. 
and further improved tissue remodelling and physiological responses via electromechanical stimulation. Furthermore, the stress-induced alignment and maturation of these tissues could be accurately simulated (Fig. 4c). Robust cardiomyocyte derivation from human induced pluripotent stem cells (iPSC-CM) has also been achieved through the canonical Wnt pathway [84] and, as such, represents an accessible and reproducible cell source that has been successfully utilised to generate myocardial tissue models. An iPSC-CM-based 3D tissue model of long QT syndrome demonstrated increased drug-induced cardiac toxicity and increased arrhythmic signs in the 3D tissue model versus the current industry standard 2D model, thus highlighting the inadequacies of current standard models for risk estimation [85].

An interesting approach to cardiac disease modelling is the combination of cardiac microtissues with microfluidic-based systems to potentially increase throughput and reduce future assay costs [87]. Such a system was utilised effectively to create a 3D pathophysiological model of Barth's syndrome, an X-linked genetic disorder characterised by cardiomyopathy, by first generating iPSC-CMs from patients with Barth's syndrome [88]. This 3D disease model was then incorporated into a microfluidic-based heart-on-a-chip platform, where the functional characteristics of the disease, such as reduced contractile stress, were accurately reproduced before correction of the disease-causing gene $T A Z$ with modRNA therapy to restore contractile function [88]. The power of in vitro pathophysiological models can be enhanced via CRISPR/ Cas9 genome editing to generate isogenic human cell line controls that reproduce disease state phenotypes, and arguably represents the next step in the field [89].

Cardiotoxicity is of major concern to myocardial tissue, with many drugs having been pulled from market because of this issue, including anticancer drugs [90], diabetes drugs, and even those targeting CVD itself [91]. To overcome this difficulty, there has been an increasing switch towards utilising human cells to create complex in vitro tissue models as platforms for drug testing. Cardiotoxicity can be induced by anthracyclines, such as doxorubicin, or more targeted therapies, such as HER2 inhibitors (e.g. trastuzumab) used for breast cancers. Furthermore, the proarrhythmic effect of drugs on cardiac tissue has been effectively modelled by Davis et al. [92]. Although there is a clear need for the utilisation of cardiac tissue for drug safety and efficacy testing, the mechanism of cardiac drug action could be determined with 3D myocardium models. For example, one of the mechanisms of action of atorvastatin was only determined through the use of a 3D myocardial tissue model in 2006, yet the drug had been utilised clinically for a decade previous to this study [93]. With increasing activity in the development of advanced therapeutics utilising approaches such as gene therapy and miRNA delivery, these novel in vitro assays could soon become one of the gold standards for safety, efficacy, and mechanistic studies for cardiac applications.

\section{State-of-the-art and future directions}

The field of tissue engineering is developing biomimetic biomaterial scaffolds that are showing increasing therapeutic potential for the repair of cardiovascular tissues. However, because of the greater correlation between these models and the physiological conditions found in the in vivo environment, a major opportunity exists to use them as in vitro 3D models for the study of cardiovascular structures and disease interventions. These in vitro models can help span the gap between $2 \mathrm{D}$ culture and in vivo testing, thus reducing the cost, time, and ethical burden of current approaches. The use of biomaterials as 3D models to facilitate greater understanding of CVD tissues can build upon other cutting-edge approaches, including miniaturised in vitro $3 \mathrm{D}$ microfluidics models, such as organs-on-a-chip, which facilitate fast repeatable results, while minimising cost and waste [90,94]. Combining multiple tissue models to create a 'body-on-a-chip system' is seen by many to be the ultimate goal to replicate the complex multiorgan crosstalk and reactions observed in vivo [95]. However, the biological complexity of these systems is often comparatively simple compared with the multi-layered and multicellular macroscale tissue-engineered constructs currently being developed in the regenerative medicine field [96]. Increased translation of native tissue complexity into the micron scale used within microfluidic platforms might allow greater correlation between these in vitro testing platforms and native tissues. However, not all testing needs to be completed in a lab-based setting and often the effect of multiple parameters can be projected using in silico simulations. A recent review in this journal profiled the work being completed using in silico models of adverse drug reactions, various protein targets, and gene expression markers to facilitate drug screening in vitro [97]. This work could be augmented and further validated using the proposed in vitro $3 \mathrm{D}$ models discussed here leading to powerful and safe drug screening methods.

Although the advantages of in vitro 3D models have been outlined here, there are situations where in vivo animal models remain indispensable, because of the shared pathogenesis and multiple manifestations of different diseases [98]. For example, Marfan Syndrome is well represented using mutagenic mouse models deficient in fibrillin 1 and these models have aided in the discovery of new drug-based treatments to curb the progression of the disease $[99,100]$; the progression of bicuspid aortic valve disease is also well replicated using mutagenic mice, which enable the natural progression of multiple pathologies, something even the most accurate in vitro 3D model of an individual cardiovascular component cannot achieve [98].

\section{Concluding remarks}

In summary, we propose that tissue-engineered biomimetic biomaterial scaffolds can not only serve a therapeutic in regenerating damaged or diseased organs and tissues, but also enable more robust testing of new technologies before in vivo testing. Increased collaboration between pharmaceutical and medical device companies with tissue-engineering researchers could see further development and validation of these models, facilitating a universally beneficial more efficient, safe, and ethical route to market for new devices, drugs, and combination products.

\section{Acknowledgements}

This work was supported by the Irish Heart Foundation (grant number 1045940) to F.J.O'B. and C.M.B., the European Research Council European Community's Seventh Framework Programme (FP7/2007-2013) under ERC grant agreement n ${ }^{\circ} 239685$ to F.J.O’B., and the Science Foundation Ireland Career Development Award (grant number 13/CDA/2119) to S.W.K. 


\section{References}

1 Mendis, S. et al. (2015) Organizational update: the world health organization global status report on noncommunicable diseases 2014; one more landmark step in the combat against stroke and vascular disease. Stroke 46, e121-e122

2 Mozaffarian, D. et al. (2015) Heart disease and stroke statistics - 2015 update: a report from the American Heart Association. Circulation 131, e29-e322

3 Nabel, E.G. and Braunwald, E. (2012) A tale of coronary artery disease and myocardial infarction. N. Engl. J. Med. 366, 54-63

4 Barron, V. et al. (2007) The effect of physiological cyclic stretch on the cell morphology, cell orientation and protein expression of endothelial cells. J. Mater. Sci. Mater. Med. 18, 1973-1981

5 Fitzgerald, K.A. et al. (2015) Life in 3D is never flat: 3D models to optimise drug delivery. J. Control. Release 215, 39-54

6 Kim, H.K. et al. (2014) Mouse models for infectious diseases caused by Staphylococcus aureus. J. Immunol. Methods 410, 88-99

7 Stingl, L. et al. (2009) 20 years of hypertension research using genetically modified animals: no clinically promising approaches in sight. ALTEX 26, 41-51

8 Mentzer, R.M. et al. (2008) Sodium-hydrogen exchange inhibition by cariporide to reduce the risk of ischemic cardiac events in patients undergoing coronary artery bypass grafting: results of the EXPEDITION study. Ann. Thorac. Surg. 85, 1261-1270

9 Flanagan, T.C. et al. (2006) Reference models for mitral valve tissue engineering based on valve cell phenotype and extracellular matrix analysis. Cells Tissues Organs 183, 12-23

10 Garciarena, C.D. et al. (2013) Sarcolemmal localisation of $\mathrm{Na}^{+} / \mathrm{H}^{+}$exchange and $\mathrm{Na}^{+}-\mathrm{HCO}_{3}{ }^{-}$co-transport influences the spatial regulation of intracellular $\mathrm{pH}$ in rat ventricular myocytes. J. Physiol. 591, 2287-2306

11 Garciarena, C.D. et al. (2013) $\mathrm{H}^{+}$-activated $\mathrm{Na}^{+}$influx in the ventricular myocyte couples $\mathrm{Ca}^{2+}$-signalling to intracellular pH. J. Mol. Cell. Cardiol. 61, 51-59

12 Bootman, M.D. et al. (2006) Calcium signalling during excitation-contraction coupling in mammalian atrial myocytes. J. Cell Sci. 119, 3915-3925

13 Louch, W. et al. (2004) Reduced synchrony of $\mathrm{Ca}^{2+}$ release with loss of T-tubules-a comparison to $\mathrm{Ca}^{2+}$ release in human failing cardiomyocytes. Cardiovasc. Res. 62, 63-73

14 Salazar, B.H. et al. (2015) Development of a cyclic strain bioreactor for mechanical enhancement and assessment of bioengineered myocardial constructs. Cardiovasc. Eng. Technol. 6, 533-545

15 Paul, S.M. et al. (2010) How to improve R\&D productivity: the pharmaceutical industry's grand challenge. Nat. Rev. Drug Discov. 9, 203-214

16 Steinberg, D. et al. (2015) Building a business model in digital medicine. Nat. Biotechnol. 33, 910-920

17 Curtin, C.M. et al. (2012) Innovative collagen nano-hydroxyapatite scaffolds offer a highly efficient non-viral gene delivery platform for stem cell-mediated bone formation. Adv. Mater. 24, 749-754

18 Ryan, A.J. and O'Brien, F.J. (2015) Insoluble elastin reduces collagen scaffold stiffness, improves viscoelastic properties, and induces a contractile phenotype in smooth muscle cells. Biomaterials 73, 296-307

19 Jana, S. et al. (2014) Scaffolds for tissue engineering of cardiac valves. Acta Biomater. 10, 2877-2893

20 Brougham, C.M. et al. (2015) Incorporation of fibrin into a collagenglycosaminoglycan matrix results in a scaffold with improved mechanical properties and enhanced capacity to resist cell-mediated contraction. Acta Biomater. 26, 205-214

21 O'Brien, F.J. et al. (2005) The effect of pore size on cell adhesion in collagen-GAG scaffolds. Biomaterials 26, 433-441

22 Wobma, H. and Vunjak-Novakovic, G. (2015) Tissue engineering and regenerative medicine 2015: a year in review. Tissue Eng. Part B: Rev. 21, 177-186

23 Wystrychowski, W. et al. (2014) First human use of an allogeneic tissue-engineered vascular graft for hemodialysis access. J. Vasc. Surg. 60, 1353-1357

24 Kelm, J.M. et al. (2012) Functionality, growth and accelerated aging of tissue engineered living autologous vascular grafts. Biomaterials 33, 8277-8285

25 Quint, C. et al. (2012) Allogeneic human tissue-engineered blood vessel. J. Vasc. Surg. 55, 790-798

26 Wise, S.G. et al. (2011) A multilayered synthetic human elastin/polycaprolactone hybrid vascular graft with tailored mechanical properties. Acta Biomater. 7, 295-303

27 Nakayama, K.H. et al. (2015) Bilayered vascular graft derived from human induced pluripotent stem cells with biomimetic structure and function. Regen. Med. 10, 745-755

28 L'Heureux, N. et al. (1998) A completely biological tissue-engineered human blood vessel. FASEB J. 12, 47-56

29 L'Heureux, N. et al. (2001) A human tissue-engineered vascular media: a new model for pharmacological studies of contractile responses. FASEB J. 15, 515-524
30 Laflamme, K. et al. (2006) Tissue-engineered human vascular media produced in vitro by the self-assembly approach present functional properties similar to those of their native blood vessels. Tissue Eng. 12, 2275-2281

31 Cardinal, K.O. and Williams, S.K. (2009) Assessment of the intimal response to a protein-modified stent in a tissue-engineered blood vessel mimic. Tissue Eng. Part A 15, 3869-3876

32 Cardinal, K.O. et al. (2006) Tissue-engineered vascular grafts as in vitro blood vessel mimics for the evaluation of endothelialization of intravascular devices. Tissue Eng. 12, 3431-3438

33 Punchard, M.A. et al. (2009) Evaluation of human endothelial cells post stent deployment in a cardiovascular simulator in vitro. Ann. Biomed. Eng. 37, 1322-1330

34 Farooq, V. et al. (2011) Restenosis: delineating the numerous causes of drugeluting stent restenosis. Circ. Cardiovasc. Interv. 4, 195-205

35 US Dept of Health and Human Sciences (2012) NHLBI Fact Book, Fiscal Year 2012. US Dept of Health and Human Sciences

36 O'Flaherty, M. et al. (2013) Contributions of treatment and lifestyle to declining CVD mortality: why have CVD mortality rates declined so much since the 1960 ? Heart 99, 159-162

37 Takaku, M. etal. (1999) An in vitro coculture model of transmigrant monocytes and foam cell formation. Arterioscler. Thromb. Vasc. Biol. 19, 2330-2339

38 Dorweiler, B. et al. (2006) A novel in vitro model for the study of plaque development in atherosclerosis. Thromb. Haemost. 95, 182-189

39 Dorweiler, B. et al. (2008) Subendothelial infiltration of neutrophil granulocytes and liberation of matrix-destabilizing enzymes in an experimental model of human neo-intima. Thromb. Haemost. 99, 373-381

40 Samady, H. et al. (2011) Coronary artery wall shear stress is associated with progression and transformation of atherosclerotic plaque and arterial remodeling in patients with coronary artery disease. Circulation 124, 779-788

41 Robert, J. et al. (2013) A three-dimensional engineered artery model for in vitro atherosclerosis research. PLOS ONE 8, e79821

42 Kim, Y. et al. (2014) Probing nanoparticle translocation across the permeable endothelium in experimental atherosclerosis. Proc. Natl. Acad. Sci. U. S. A. 111, 1078-1083

43 D'Arcy, J.L. et al. (2011) Valvular heart disease: the next cardiac epidemic. Heart 97, 91-93

44 Butcher, J.T. et al. (2011) Aortic valve disease and treatment: the need for naturally engineered solutions. Adv. Drug Deliv. Rev. 63, 242-268

45 Jana, S. et al. (2015) Cells for tissue engineering of cardiac valves. J. Tissue Eng. Regen. Med. http://dx.doi.org/10.1002/term.2010

46 Tremblay, C. et al. (2014) A new construction technique for tissue-engineered heart valves using the self-assembly method. Tissue Eng. Part C: Methods 20, 905-915

47 Reimer, J.M. et al. (2015) Pediatric tubular pulmonary heart valve from decellularized engineered tissue tubes. Biomaterials 62, 88-94

48 Weber, B. et al. (2013) Off-the-shelf human decellularized tissue-engineered heart valves in a non-human primate model. Biomaterials 34, 7269-7280

49 Masoumi, N. et al. (2014) Tri-layered elastomeric scaffolds for engineering heart valve leaflets. Biomaterials 35, 7774-7785

50 Moreira, R. et al. (2014) TexMi: development of tissue-engineered textilereinforced mitral valve prosthesis. Tissue Eng. Part C: Methods 20, 741-748

$51 \mathrm{Liu}$, A.C. et al. (2007) The emerging role of valve interstitial cell phenotypes in regulating heart valve pathobiology. Am. J. Pathol. 171, 1407-1418

52 Butcher, J.T. et al. (2006) Transcriptional profiles of valvular and vascular endothelial cells reveal phenotypic differences: influence of shear stress. Arterioscler. Thromb. Vasc. Biol. 26, 69-77

53 Schoen, F.J. (2008) Evolving concepts of cardiac valve dynamics: the continuum of development, functional structure, pathobiology, and tissue engineering. Circulation 118, 1864-1880

54 Chester, A.H. and Taylor, P.M. (2007) Molecular and functional characteristics of heart-valve interstitial cells. Philos. Trans. R. Soc. Lond. B: Biol. Sci. 362, 1437-1443

55 Liu, A.C. and Gotlieb, A.I. (2008) Transforming growth factor- $\beta$ regulates in vitro heart valve repair by activated valve interstitial cells. Am. J. Pathol. 173, 1275-1285

56 Benton, J.A. et al. (2009) Characterization of valvular interstitial cell function in three dimensional matrix metalloproteinase degradable PEG hydrogels. Biomaterials 30, 6593-6603

57 Kloxin, A.M. et al. (2010) In situ elasticity modulation with dynamic substrates to direct cell phenotype. Biomaterials 31, 1-8

58 Wyss, K. et al. (2012) The elastic properties of valve interstitial cells undergoing pathological differentiation. J. Biomech. 45, 882-887

59 Yip, C.Y.Y. et al. (2009) Calcification by valve interstitial cells is regulated by the stiffness of the extracellular matrix. Arterioscler. Thromb. Vasc. Biol. 29, 936-942 
60 Masoumi, N. et al. (2013) Valvular interstitial cell seeded poly(glycerol sebacate) scaffolds: toward a biomimetic in vitro model for heart valve tissue engineering. Acta Biomater. 9, 5974-5988

61 Hasan, A. et al. (2014) Biomechanical properties of native and tissue engineered heart valve constructs. J. Biomech. 47, 1949-1963

62 Schoen, F.J. (1997) Aortic valve structure-function correlations: role of elastic fibers no longer a stretch of the imagination. J. Heart Valve Dis. 6, 1-6

63 Tseng, H. et al. (2014) A three-dimensional co-culture model of the aortic valve using magnetic levitation. Acta Biomater. 10, 173-182

64 Puperi, D.S. et al. (2015) 3-Dimensional spatially organized PEG-based hydrogels for an aortic valve co-culture model. Biomaterials 67, 354-364

65 Flanagan, T.C. et al. (2006) A collagen-glycosaminoglycan co-culture model for heart valve tissue engineering applications. Biomaterials 27, 2233-2246

66 Richards, J. et al. (2013) Side-specific endothelial-dependent regulation of aortic valve calcification. Am. J. Pathol. 182, 1922-1931

67 Hjortnaes, J. et al. (2013) Simulating early calcific aortic valve disease within novel in vitro 3D tissue platform. Eur. Heart J. 34, 3908

68 Kamel, P.I. et al. (2014) Metabolic regulation of collagen gel contraction by porcine aortic valvular interstitial cells. J. R. Soc. Interface 11, 20140852

69 Gould, R.A. et al. (2012) Cyclic strain anisotropy regulates valvular interstitial cell phenotype and tissue remodeling in three-dimensional culture. Acta Biomater. 8, $1710-1719$

70 Butcher, J.T. and Nerem, R.M. (2006) Valvular endothelial cells regulate the phenotype of interstitial cells in co-culture: effects of steady shear stress. Tissue Eng. 12, 905-915

71 Garciarena, C.D. et al. (2015) Coordinated molecular cross-talk between Staphylococcus aureus, endothelial cells and platelets in bloodstream infection. Pathogens 4, 869-882

72 Nejad, S.P. et al. (2015) Biomechanical conditioning of tissue engineered heart valves: too much of a good thing? Adv. Drug Deliv. Rev. 96, 161-175

73 Brougham, C.M. et al. (2014) A new reinforced fibrin collagen glycosaminoglycan material to resist tissue retraction in heart valves. World Congress of Biomechanics

$74 \mathrm{Li}$, C. et al. (2013) The progression of calcific aortic valve disease through injury, cell dysfunction, and disruptive biologic and physical force feedback loops. Cardiovasc. Pathol. 22, 1-8

75 Rothman, R.B. et al. (2000) Evidence for possible involvement of 5-HT2B receptors in the cardiac valvulopathy associated with fenfluramine and other serotonergic medications. Circulation 102, 2836-2841

76 Garg, V. et al. (2005) Mutations in NOTCH1 cause aortic valve disease. Nature 437, 270-274

77 MacGrogan, D. et al. (2011) Notch signaling in cardiac valve development and disease. Birth Defects Res. A: Clin. Mol. Teratol. 91, 449-459

78 Connell, P.S. et al. (2015) Regurgitation hemodynamics alone cause mitral valve remodeling characteristic of clinical disease states in vitro. Ann. Biomed. Eng. 44, 954-967

79 Farrar, E.J. et al. (2015) Endothelial-derived oxidative stress drives myofibroblastic activation and calcification of the aortic valve. PLOS ONE 10, e0123257

80 Force, T. and Kolaja, K.L. (2011) Cardiotoxicity of kinase inhibitors: the prediction and translation of preclinical models to clinical outcomes. Nat. Rev. Drug Discov. $10,111-126$
81 Tiburcy, M. et al. (2011) Terminal differentiation, advanced organotypic maturation, and modeling of hypertrophic growth in engineered heart tissue. Circ. Res. 109, 1105-1114

82 Boudou, T. et al. (2012) A microfabricated platform to measure and manipulate the mechanics of engineered cardiac microtissues. Tissue Eng. Part A 18, 910-919

83 Thavandiran, N. et al. (2013) Design and formulation of functional pluripotent stem cell-derived cardiac microtissues. Proc. Natl. Acad. Sci. U. S. A. 110 E4698-E4707

84 Lian, X. et al. (2012) Robust cardiomyocyte differentiation from human pluripotent stem cells via temporal modulation of canonical Wnt signaling. Proc. Natl. Acad. Sci. U. S. A. 109, E1848-E1857

$85 \mathrm{Ma}$, Z. et al. (2014) Three-dimensional filamentous human diseased cardiac tissue model. Biomaterials 35, 1367-1377

86 van Spreeuwel, A.C.C. et al. (2014) The influence of matrix (an)isotropy on cardiomyocyte contraction in engineered cardiac microtissues. Integr. Biol. (Camb.) 6, 422-429

87 Agarwal, A. et al. (2013) Microfluidic heart on a chip for higher throughput pharmacological studies. Lab Chip 13, 3599-3608

88 Wang, G. et al. (2014) Modeling the mitochondrial cardiomyopathy of Barth syndrome with induced pluripotent stem cell and heart-on-chip technologies Nat. Med. 20, 616-623

89 Grobarczyk, B. et al. (2015) Generation of isogenic human iPS cell line precisely corrected by genome editing using the CRISPR/Cas9 system. Stem Cell Rev. 11, 774-787

90 Ewer, M.S. and Ewer, S.M. (2010) Cardiotoxicity of anticancer treatments: what the cardiologist needs to know. Nat. Rev. Cardiol. 7, 564-575

91 Shah, R.R. (2006) Can pharmacogenetics help rescue drugs withdrawn from the market? Pharmacogenomics 7, 889-908

92 Davis, R.P. et al. (2012) Cardiomyocytes derived from pluripotent stem cells recapitulate electrophysiological characteristics of an overlap syndrome of cardiac sodium channel disease. Circulation 125, 3079-3091

93 Mühlhäuser, U. et al. (2006) Atorvastatin desensitizes beta-adrenergic signaling in cardiac myocytes via reduced isoprenylation of G-protein gamma-subunits. FASEB J. 20, 785-787

94 Zhang, Y.S. et al. (2015) From cardiac tissue engineering to heart-on-a-chip: beating challenges. Biomed. Mater. 10, 34006

95 Eisenstein, M. (2015) Artificial organs: honey, I shrunk the lungs. Nature 519, S16-S18

96 Foster, G.A. et al. (2013) On-chip phenotypic analysis of inflammatory monocytes in atherogenesis and myocardial infarction. Proc. Natl. Acad. Sci. U. S. A. 110, 13944-13949

97 Ivanov, S.M. et al. (2015) In silico assessment of adverse drug reactions and associated mechanisms. Drug Discov. Today 21, 58-71

98 Krishnamurthy, V.K. et al. (2014) Review of molecular and mechanical interactions in the aortic valve and aorta: implications for the shared pathogenesis of aortic valve disease and aortopathy. J. Cardiovasc. Transl. Res. 7, 823-846

99 Ramirez, F. and Dietz, H.C. (2007) Marfan syndrome: from molecular pathogenesis to clinical treatment. Curr. Opin. Genet. Dev. 17, 252-258

100 Habashi, J.P. et al. (2006) Losartan, an AT1 antagonist, prevents aortic aneurysm in a mouse model of Marfan syndrome. Science 312, 117-121 